\title{
Migración transfronteriza y trabajo infantil en el Soconusco, Chiapas
}

\author{
LAURA ITZEL RAMÍREZ RAMOS*, EMMA ZAPATA MARTELO**, \\ VERÓNICA VÁZQUEZ GARCÍA***, DOMINGA AUSTREBERTA \\ NAZAR BEUTELSPACHER ${ }^{* * * *}$, NAIMA CÁRCAMO TOALÁ*****
}

RESUMEN: En este trabajo se analizan las características del trabajo infantil en las fincas cafetaleras del Soconusco, Chiapas, México. Históricamente, esta región ha sido un importante mercado de trabajo para familias migrantes provenientes de Guatemala, cuyas condiciones de vida les obligan a ocupar puestos laborales que los exponen a ser víctimas, entre otras cosas, de explotación y de violación tácita de los derechos humanos. Se problematiza la división genérica del trabajo en las ocupaciones y responsabilidades en los ámbitos productivo y reproductivo, y las razones por las que niños, niñas y adolescentes migrantes transfronterizos se incorporan al trabajo productivo en las fincas. El trabajo infantil beneficia directamente al capital, a la vez que existe una falta de responsabilidad de los gobiernos para desarrollar políticas efectivas de combate a la pobreza y crear mecanismos eficientes de supervisión y protección de los derechos humanos y laborales.

PALABRAS CLAVE: trabajo infantil, migración transfronteriza, Chiapas, derechos humanos, vulnerabilidad social.

* Estudiante de la Maestría en Desarrollo Rural del Colegio de Postgraduados, Estado de México, México.

** Profesora Investigadora del Programa de Desarrollo Rural del Colegio de Postgraduados en Ciencias Agrícolas, Estado de México, México.

*** Profesora investigadora del Programa de Desarrollo Rural del Colegio de Postgraduados en Ciencias Agrícolas, Estado de México, México.

**** Investigadora del Departamento Sociedad, Cultura y Salud de El Colegio de la Frontera Sur, Chiapas, México.

***** Investigadora de El Colegio de la Frontera Sur, Chiapas, México, y coordinadora operativa del Proyecto Salud Mesoamérica 2015, Nicaragua. 
ABSTRACT: This work analyzes the characteristics of child labor in the coffee estates of the Soconusco region, Chiapas, Mexico. Historically, this region has been an important labor market for migrant families coming from Guatemala, whose quality of life leads them to take up labor roles that put them at risk of being victims, among other things, of labor exploitation and the tacit violation of their human rights. It examines the gender division of labor in the jobs and responsibilities both in areas of production and reproduction, and the reasons why cross-border migrant children - boys and girlsand teenagers, are incorporated into the plantation's productive work. Child labor directly benefits capital in a context where the responsibility of governments to develop effective policies to combat poverty and create efficient mechanisms for the supervision and protection of human and labor rights is lacking.

KEY WORDS: child labor, cross-border migration, Chiapas, human rights, social vulnerability. 


\section{INTRODUCCIÓN}

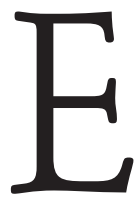

n la lógica de la producción de mercancías, la maximización y acumulación de capital a costa de la mano de obra barata, la explotación de recursos naturales, la precarización y privatización de servicios sociales, se coloca a las y los individuos ante escenarios donde la violación de los derechos humanos es recurrente. Pierden su calidad de personas, se convierten en objetos cuya utilidad es condicionada por los mercados y su existencia es fácilmente reemplazable (George, 2003; Pogge, 2007; Feldmann, 2008; Gzesh, 2008; Branka y Virik, 2011; Márquez y Delgado, 2011).

La migración laboral —individual o familiar — es una estrategia de supervivencia que las personas utilizan para hacer frente a las condiciones impuestas por el sistema capitalista (López, 2011). En la frontera sur de México, personas de origen guatemalteco, provenientes de los departamentos colindantes a ésta (en su mayoría), se insertan en el mercado laboral de las fincas cafetaleras del Soconusco. Una de las consecuencias de este fenómeno migratorio es la migración y el trabajo infantil, en donde niños, niñas y adolescentes constituyen una importante fuente de mano de obra barata con impactos trascendentales en su integridad física, social y psicológica (Casillas, 2010).

Según el Centro de Estudios Migratorios de la Unidad de Política Migratoria de la Secretaría de Gobernación (Segob) (2012), durante 2012 se extendieron 21,743 Tarjetas de Visitantes Trabajadores Fronterizos en Chiapas, de los cuales $99.9 \%$ se emplearían en el sector agrícola y sólo $0.1 \%$ en el sector de servicios. De éstos, $14.6 \%$ eran para adolescentes de 16 a 19 años. ${ }^{1}$ En este artículo se describen las características del trabajo infantil en las fincas cafetaleras del Soconusco, Chiapas, con el fin de conocer los procesos de incorporación en la migración transfronteriza laboral. Se pretende responder a dos preguntas específicas: ¿̇cuáles son las condiciones laborales de niños, niñas y adolescentes en las fincas cafetaleras?, y żqué actividades realizan en los ámbitos productivo y reproductivo?

${ }^{1}$ En la emisión de estas tarjetas no se incluye a los menores de 16 años pues no son reconocidos como trabajadores formales ante la ley. 


\section{EL CONTEXTO: LA MIGRACIÓN TRANSFRONTERIZA EN LA FRONTERA SUR}

Guatemala ha sufrido los embates de fuertes crisis climatológicas y financieras y de violencia social. ${ }^{2}$ La insuficiencia de acciones orientadas a la agricultura de autoabasto y al desarrollo rural, la carencia de un sistema de protección social público que provea un seguro de desempleo, la pérdida de puestos de trabajo y la disminución en la disponibilidad de recursos económicos en el hogar han afectado de manera severa el desarrollo y las condiciones de vida de la población (UNICEF, 2010).

Datos de la Encuesta Nacional de Condiciones de Vida (encovi, 2011) muestran que $53.7 \%$ de las y los guatemaltecos viven en condiciones de pobreza, lo cual se manifiesta en su limitado acceso a la seguridad alimentaria. Entre los sectores más desprotegidos, debido a la brecha existente entre el costo de la canasta básica y el salario mínimo, se encuentran las y los jornaleros agrícolas, quienes debido a la baja capacidad productiva tienen menor posibilidad de acceder a los alimentos. Sólo 19.1\% de los hogares de Guatemala pueden considerarse seguros desde el punto de vista alimentario (FAO, 2011; ENCOVI, 2011).

De acuerdo con la Organización Internacional para las Migraciones (OIM) y el Fondo de las Naciones Unidas para la Infancia (UNICEF, 2010), la migración de las y los guatemaltecos se realiza con dos finalidades fundamentales: para buscar trabajo (37.2\%) y para mejorar sus condiciones económicas (51.7\%). ${ }^{3}$ Los flujos migratorios se encuentran constituidos, principalmente,

2 Los huracanes Mitch, Stand y la tormenta tropical Agatha afectaron de manera considerable su territorio, provocando la pérdida de viviendas, carreteras y cultivos agrícolas. En el caso de la crisis financiera en 2008, el PIB sólo manifestó $0.6 \%$ de crecimiento, lo cual afectó seriamente la economía de las y los guatemaltecos (UNICEF, 2010). La violencia social no ha dejado de ser una problemática, en tres años (2007-2011) se registraron 29,723 casos de muertes violentas, de las cuales 8.0\% (2,293) eran niños y niñas menores de 17 años, $83.0 \%$ de estas muertes se realizaron con arma de fuego (PDH, 2011).

${ }^{3}$ La categoría otros (11.1\%) es secundaria e incluye razones como reunificación familiar, la construcción de viviendas, conflictos familiares y sociales, desastres naturales, entre otras.

$42 \frac{\text { PRIMER SEMESTRE } 2014}{\text { MIGRACIÓN Y DESARROLLO NÚM. } 22}$ 
por personas jóvenes; $64.7 \%{ }^{4}$ de quienes migran está ubicados entre los 7 y 29 años de edad, mientras que el resto (35.3\%) son mayores de 30 años. Los migrantes ven afectadas sus posibilidades de desarrollo y sufren violaciones a sus derechos laborales y humanos (Cruz y Rojas, s/f; Liwski, 2012; Zapata y Suárez, 2012).

La migración de guatemaltecos tiene como principal finalidad arribar a Estados Unidos; sin embargo, existe un importante flujo de migrantes que se insertan en áreas agrícolas laborales de la frontera sur de México, considerada uno de los principales lugares de destino o tránsito de miles de personas centroamericanas que de manera documentada o indocumentada van en busca de oportunidades laborales que mejoren o aseguren sus condiciones de vida (Palma, 2007).

Entre las regiones socioeconómicas que integran este territorio, el Soconusco ha sido, históricamente, por la producción de café, un espacio que requiere de la mano de obra de muchas y muchos trabajadores, por lo cual se ha convertido en un importante mercado de trabajo y polo de atracción de migrantes (Anguiano, 2008; Castillo, 2006, 2009). Al ser una migración de tipo familiar, la de origen guatemalteco constituye una práctica generacional donde la niñez migrante se inserta en condiciones laborales extremas y vejatorias. Girón (2010) y Casillas (2010) mencionan que estos infantes son una importante fuente de mano de obra barata y especializada sólo reconocida a través de la mano de obra adulta y del trabajo familiar que acepta y acata roles de subordinación y servilismo.

\section{Propuesta conceptual: trabajo infantil Y DIVISIÓN GENÉRICA DE TRABAJO}

La definición del trabajo infantil ha generado una polémica importante debido a que existe dificultad al delimitarlo, conceptualizarlo y, por lo tanto,

${ }^{4}$ De estos, $0.1 \%$ son niños y niñas de entre 7 y 9 años; $0.8 \%$ tienen de 10 a 14 años y $16.8 \%$ son adolescentes entre los 15 y 19 años. 
al establecer líneas de acción para su tratamiento (Saadeh, 2011). Entre la polisémica variedad de acepciones se pueden encontrar aquéllas que lo consideran como un proceso benéfico para la socialización de niños y niñas, hasta las que refieren una grave violación a los derechos básicos de la infancia (Krichesky, 1992; Barreiro, 1998).

El trabajo infantil para Krichesky (citado por Galeana, 2000) constituye una "estrategia de supervivencia» ante condiciones de marginación social. Brizzio (1996) coincide al definirlo como las actividades que realizan niños y niñas de familias pobres presionadas por la necesidad de obtener una remuneración (en dinero o especie). Estas actividades se alejan de objetivos socializadores y tienen un carácter discriminatorio, pues se desarrollan bajo características de invisibilidad. Las familias se transforman en pequeñas unidades autoexplotadoras que permiten la reproducción del sistema capitalista de producción. Se disfraza el trabajo infantil haciéndolo pasar como una práctica cultural de cooperación y solidaridad familiar (Glokner, 2010). Sin embargo, las causas del trabajo infantil no sólo están del lado de las familias, sino también de los empleadores que han hecho uso extensivo de esta mano de obra (Rojas, 2006; Cos, 1998). El trabajo infantil beneficia directamente al capital debido a que cubre la parte no pagada del trabajo del obrero y conlleva a la sobreexplotación y la depresión salarial (Brizzio, 2002; Sánchez y Macchia, 2002 citado por Rojas, 2006).

La perspectiva de género permite distinguir entre distintos tipos de trabajo y determinar los beneficios que cada integrante de la familia deriva de sus actividades. La división genérica del trabajo organiza, segmenta y jerarquiza las ocupaciones y responsabilidades de dos ámbitos: el productivo y el reproductivo, lo cual afecta de manera diferenciada y en distintos niveles el tiempo, salarios, recursos, autonomía, reconocimiento social, calidad de vida y acceso a oportunidades de hombres y mujeres (Carrasquer, Torns, Tejero y Romero, 1998; Martínez, Martínez, Barrientos y Paredes, 2003; Todaroy Yáñez, 2004; Moreno, 2007; Suárez, Zapata, Ayala, Cárcamo y Manjarrez, 2011).

El trabajo productivo es el que genera ingresos a través de la fabricación u oferta de bienes y servicios. Generalmente se realiza en la esfera pública. Por su parte, el trabajo reproductivo engloba todas las actividades de cuidado 
y gestión de la familia. Se realiza sin recibir una remuneración económica y se lleva a cabo predominantemente en el espacio privado, aunque también tiene injerencia en el espacio público. Es fundamental en el desarrollo humano y contribuye a liberar a algunos miembros de cargas laborales reproductivas para realizar más trabajo productivo. Ha sido asignado a las mujeres en la mayoría de las sociedades del mundo. No es reconocido ni valorado como importante, se minimiza su utilidad e incluso las personas que lo llevan a cabo lo consideran como un conjunto de actividades que no requiere conocimientos, habilidades, ni aprendizajes específicos (Carrasquer et al., 1998; Delson, 2003; Martínez et al., 2003; Moreno, 2007).

La migración y el trabajo infantil productivo y reproductivo constituyen factores de vulnerabilidad ${ }^{5}$ que tiene la particularidad de limitar la realidad de las y los individuos, librándolos (as) a su propia suerte en una economía de mercado. Los niños y niñas pierden la posibilidad real de crear un proyecto de vida futuro, sano, humano y digno (Brasesco y Mendoza, 2011; Rojas, 2012).

\section{Metodología de trabajo}

Los datos fueron recolectados en tres fincas cafetaleras del estado de Chiapas, de enero a marzo de 2012. La elección de las fincas se llevó a cabo tomando en cuenta el número de hectáreas de producción, su estado de certificación, la existencia de personal extranjero (de origen guatemalteco) contratado como jornalero(a) agrícola, el tipo de contratación de las y los trabajadores y la accesibilidad de los dueños y administradores para realizar el estudio.

Se utilizó un enfoque metodológico mixto con técnicas cuantitativas, cualitativas y participativas. En primera instancia se aplicó una encuesta no

${ }^{5}$ La vulnerabilidad se origina a partir de la coexistencia de factores internos (edad, sexo, estado de salud, origen étnico, discapacidad, orientaciones sexuales, constitución física) y externos (nivel de ingresos, falta de empleo, crisis económicas, condición migratoria, falta de políticas públicas adecuadas para la atención de una problemática social) que al combinarse disminuyen la capacidad que tiene una persona, grupo o comunidad para enfrentar una situación determinada (Salgado, González, Bojorquez e Infante, 2007; CNDH, 2009). 
probabilística a 129 familias, a través de la cual se recabó información de 187 niños y niñas de 0 a 17 años. También se hicieron 22 entrevistas con distintos actores sociales (un representante del gobierno de Guatemala, representantes de organismos defensores de los derechos humanos, directores municipales y estatales de oficinas de atención a migrantes, responsables de programas de salud, educación y protección contra el trabajo infantil). Finalmente, se realizaron seis talleres con niños, niñas y adolescentes de 5 a 17 años para que compartieran sus experiencias laborales. La observación participante fue utilizada de manera constante y sistemática.

\section{El trabajo infantil en las fincas cafetaleras del Soconusco}

México es uno de los mayores productores de café en el mundo, ocupa el sexto lugar como productor y el undécimo como exportador. Esta actividad genera ingresos en divisas por aproximadamente 900 millones de dólares (Sagarpa, 2012). En 2001, el Soconusco contaba con 15,043 productores y una superficie cultivada de 77,123 hectáreas, lo que representa la zona de mayor importancia en la producción del grano (Censo Cafetalero del Inmecafé 1992 citado por CEFP, 2001). La producción de café requiere de grandes cantidades de mano de obra especializada durante todo su ciclo.

Una encuesta realizada por el Instituto para la Seguridad y la Democracia (INSYDE, 2008) muestra que $80.0 \%$ de las personas en Tapachula declaraba que la migración de guatemaltecos a México beneficia a su municipio por dos motivos fundamentales: 1) toman los trabajos que ningún mexicano haría, y 2) aceptan bajos salarios. Dice el vicecónsul de Guatemala:

la mano de obra guatemalteca para la región tapachulteca es muy, muy importante, sin ella la región no va a producir, hay que tomar en cuenta que la mano de obra en las fincas cafetaleras no la puede dar cualquiera [...] [,] es gente que tiene la especialidad en cuanto al manejo y corte del café, entonces esa mano de obra no la van a conseguir en Tapachula, además al empleador mexicano le va a salir mucho más económico, un empleado mexicano no va ir a trabajar por

$46 \frac{\text { PRIMER SEMESTRE } 2014}{\text { MIGRACIÓN Y DESARROLLO NÚM. } 22}$ 
cincuenta pesos; sin embargo, muchos guatemaltecos vienen a trabajar por el salario mínimo (Vicecónsul de Guatemala, febrero, 2012).

La «ayuda» de niños, niñas y adolescentes dentro de los cafetales no es considerada una violación a las leyes referentes al trabajo infantil por los dueños y administradores de las fincas. Argumentan que son las y los jefes de familia «quienes les exigen a los hijos que trabajen para cubrir sus necesidades económicas» (Administrador de la finca «I», febrero de 2012). Este discurso es aceptado también institucionalmente por los responsables de la supervisión de los centros de trabajo.

[...] nosotros no podemos tomar acciones porque no está recibiendo un salario el niño, sino que está aprendiendo y esto es parte del mismo juego de la forma de vida y de aprender a vivir, cuántos niños no aprendimos hacer cosas así o jugamos a que éramos abogados o doctores y terminamos siendo abogados y doctores [...] (Inspector del trabajo, febrero de 2012).

Desde esta lógica, el trabajo infantil, además de criminalizar a las víctimas (Saadeh, 2011), no visibiliza a la niñez ni a sus familias y se niega a entender el fenómeno desde otras perspectivas, como la de la economía familiar o comunitaria, el incumplimiento de sus derechos humanos y la falta de oportunidades productivas que posibiliten mejores condiciones socioeconómicas. Esta posición institucional deja invisible la responsabilidad de los gobiernos ante la ausencia de políticas efectivas de combate a la pobreza y la falta de mecanismos eficientes de supervisión y protección de los derechos laborales. Además, condona la violación a los derechos de las y los trabajadores jornaleros por las empresas cafetaleras. En este aspecto se comparte la perspectiva de Saadeh (2011) cuando cita a Eduardo Galeano: «se culpa al pobre y se deja impune al sistema que lo crea».

Con estas argumentaciones se ignoran los impactos y repercusiones en todos los ámbitos de la vida de los infantes: grandes esfuerzos físicos que afectan su desarrollo, la falta de una remuneración económica directa, la obligación de adquirir nuevas responsabilidades y roles económicos o de 
cuidado al interior de las familias. Además, la interferencia de manera contundente del pleno acceso y ejercicio de sus derechos; por ejemplo, el derecho a la educación, a la salud, a recibir una alimentación adecuada, al esparcimiento y al descanso. Las y los individuos pierden la posibilidad de desarrollar capacidades que les permitan responder a situaciones de riesgo o generar acciones que les posibiliten acceder a mejores condiciones de vida. Es decir, los inserta en un estado de alta vulnerabilidad social.

\section{Condiciones laborales}

Niños y niñas tienen cuatro opciones mientras están en las fincas: 1) realizar los diferentes trabajos reproductivos del grupo doméstico mientras sus padres trabajan; 2) asistir a la escuela (si es que existe dentro de las fincas), esta posibilidad no los exime de cumplir con sus responsabilidades de trabajo reproductivo, pero sí evita y retarda su proceso de inserción en trabajos productivos; 3) "acompañar» a sus padres y madres a realizar los diversos trabajos productivos relacionados con el café, donde cumplen con obligaciones laborales, viven las extenuantes y precarias condiciones de trabajo y no tienen una remuneración económica directa por su trabajo, y 4) los mayores de 14 años pueden insertarse como trabajadores y trabajadoras directas de las fincas, con un sueldo menor y bajo el cuidado y responsabilidad de los jefes y jefas de familia.

Se identificaron cuatro razones por las que niños y niñas trabajan en las fincas cafetaleras: 1) la obligación, pues se encuentran subordinados a las personas adultas, por lo que los trabajos que realizan, no se cuestionan: "yo ayudo porque es mi papá», "es mi obligación, ya que ellos de pequeña me cuidaron»; 2) la solidaridad, existen casos de niños y niñas que trabajan productiva y reproductivamente con la finalidad de disminuir las cargas laborales de los padres y madres: «le ayudo para no ver cansada, ni sufriendo a mi mamá $[. .$.$] », «es mi mamá y ella no tiene que hacer lo que hacen los hom-$ bres»; 3) el gusto, se encontraron casos de niñas, principalmente, que respondían que ayudaban a sus padres porque les "gustaba». Esta respuesta debe

$48 \frac{\text { PRIMER SEMESTRE } 2014}{\text { MIGRACIÓN Y DESARROLLO NÚM. } 22}$ 
ser observada como una consecuencia de la adaptación a un medio en donde los roles sociales de género y de edad se encuentran establecidos y determinados. Es una señal de la falta de opciones y oportunidades para decidir entre referentes o actividades diferentes. El gusto, no es gusto si es por obligación: "me gusta porque sólo trabajamos y somos muchos», y 4) la percepción de su propia conveniencia, en el grupo de adolescentes varones se refirió que ellos trabajan para contar con sus propios recursos económicos: «así me conviene», "para aprender a trabajar», "para mí», "para que me alcance el dinero», "para tener dinero», "para trabajar» (Talleres «Un día en mi vida», febrero-marzo de 2012).

El $68.5 \%(n=187)$ de niños, niñas y adolescentes realizan trabajos productivos o reproductivos en las fincas. Quienes no lo hacen, es porque todavía no cuentan con las condiciones físicas para poder llevarlos a cabo (niños y niñas menores de cinco años). Las y los jefes de familia refirieron que 79.7\% ( $n=187)$ de sus hijos e hijas apoyan más a sus familias cuando están en las fincas que cuando permanecen en sus lugares de origen. Los principales motivos para valorar de esta manera su «ayuda» son: "porque allá no hay trabajo para chamacos como aquí», "ayudan y los vemos más», "ayudan mientras uno trabaja», "me ayudan y es el único lugar donde pueden trabajar», «aquí le dan su trabajito», "porque ayuda cuando los demás trabajamos», «porque de aquí se llevará unos centavitos más», "porque cuida al bebé en la mañana». En este contexto, niños, niñas y adolescentes no son «aprendices» ni «ayudantes» y mucho menos «acompañantes» de las y los jefes de familia, son el remplazo de la mamá en los trabajos reproductivos y el complemento en los trabajos productivos realizados por ambos padres.

Las condiciones laborales bajo las cuales se desarrolla el trabajo infantil dentro de las fincas se caracterizan por cambios bruscos de temperatura (en un mismo día puede haber lluvias, humedad y calor) y el uso de instrumentos peligrosos de trabajo (regularmente herramientas punzocortantes); la necesidad de caminar largas distancias, reconocer caminos, administrar el tiempo, caminar sobre terrenos lodosos y empinados, esquivar ramas, evitar resbalones, aguantar piquetes de mosquitos u otros insectos, subir y bajar árboles, cargar grandes cantidades de café (costales de entre 40 y $60 \mathrm{~kg}$, excesivos 
para la estatura y estado de nutrición de las y los niños migrantes); además de una alimentación deficiente con dos tiempos constituidos por frijoles, tortillas y café (alimentos que los padres y madres costean o comparten con sus hijos e hijas ya que éstos no son reconocidos como jornaleros o ayudantes, motivo por el que no pueden acceder a este derecho), precarias condiciones de vivienda, hacinamiento, aislamiento, carencia de actividades recreativas y educativas integrales, etcétera.

Estas características insertan a la infancia migrante en condiciones de vulnerabilidad, tensión, incertidumbre y precarización. El 32.1\% de las y los encuestados mencionan que sus hijos e hijas sufren a causa, principalmente, de las condiciones laborales, porque: «a veces se acaba el dinero y no tiene uno para la comida», "porque el trabajo es todos los días, se madruga, es peligroso y la comida no es buena». Ante este escenario, la única opción para las familias y, por lo tanto, para la niñez es buscar aquellos lugares de trabajo en donde los traten «menos mal»: «nadie los obliga a trabajar, al que le gustó trabajar aquí, regresa por sí sólo [...]» (Mayordomo de finca, febrero de 2012). De Sousa (2006) referiría a estas poblaciones como inmersas en el fascismo social de la seguridad, en dónde las y los trabajadores se insertan a estos procesos con la finalidad de asegurar condiciones mínimas de subsistencia.

\section{División GENÉRICA DEL TRABAJO}

La división genérica del trabajo organiza la dinámica interna y externa de las familias dentro de las fincas. Se encontró que 45.7\% de las niñas referidas por los padres realizan actividades reproductivas, mientras que la participación de los niños en éstas sólo corresponde a 20.8\%. La situación se invierte cuando se contemplan los trabajos realizados fuera del hogar, es decir, el trabajo en campo o trabajos productivos, en donde los niños suelen tener una mayor frecuencia (27.4\% frente a $4.9 \%$ de las mujeres).

Hay dos espacios donde cotidianamente se realiza el trabajo infantil: el público, que comprende los campos de cosecha, patios de producción, arroyos, lavaderos, baños, canchas de futbol, iglesias, escuelas, tiendas, oficinas 
administrativas y espacios médicos, y el espacio privado, que corresponde a las galleras (lugares de descanso dentro de las fincas) y cocinas. Cada uno de éstos cuenta con sus respectivas limitantes y reglas de acceso, uso y control de recursos, espacios y tiempos.

\section{Trabajo productivo}

Dentro de las fincas cafetaleras, el trabajo productivo se identifica con actividades relacionadas a la siembra, mantenimiento y cosecha del café. Este tipo de trabajo puede ser remunerado o no remunerado directamente, de acuerdo con las edades de quienes lo realizan. Su pago es individual, si quien lo efectúa es mayor de 14 años, o familiar, si son niños y niñas menores de 14. La determinación de esta condición se da a partir de lo dispuesto en el artículo 22 de la Ley Federal del Trabajo que a la letra dice:

Queda prohibida la utilización del trabajo de los menores de catorce años y de los mayores de esta edad y menores de dieciséis que no hayan terminado su educación obligatoria, salvo los casos de excepción que apruebe la autoridad correspondiente en que a su juicio haya compatibilidad entre los estudios y el trabajo.

No se contrata a niños y niñas menores de 14 años pero no se impide que trabajen garantizando su pleno acceso a otros derechos como el educativo, esparcimiento y descanso. Respecto a los mayores de 14 y menores de 16 tampoco se sigue la norma, pues no se comprueba que hayan concluido su educación primaria y secundaria, además de que no existe una compatibilidad entre los trabajos realizados en la finca, su condición migratoria y los estudios. Ante este argumento jurídico, niños, niñas y adolescentes quedan expuestos y desprotegidos a través de un discurso que violenta sus derechos.

El trabajo productivo se realiza con mayor frecuencia a partir de los 12 años, donde $60.0 \%$ de las y los adolescentes de 12 a 17 años $(n=40)$ se dedican de manera explícita a trabajar con los jefes y jefas de familia en la producción 
del café. Se reconoce un número mayor de varones realizando estas actividades (69.6\% sobre $30.4 \%$ de mujeres). Es importante anotar que los varones de este grupo de edad no se involucran en la realización de trabajos reproductivos. Las mujeres adolescentes, a diferencia de ellos, además de realizar trabajos productivos, no se eximen de realizar los de tipo reproductivo.

La participación de los niños (as) de 5 a 11 en el trabajo productivo es menor, sólo $19.2 \%(n=26)$ de este grupo lo hacen de manera exclusiva y se especializan en la tapisca (corte de café), el cajete (realización de zanjas para el cultivo de café), limpieza general de la finca (recolección de basura), la pepena (recolección de los últimos granos de café), entre otros. Cubren doble jornada laboral $11.5 \%$ de ellos y ellas, es decir, realizan trabajos productivos pero también reproductivos.

Los salarios de las personas adultas dentro de las fincas no son mayores a 100.00 pesos diarios, dependiendo del tipo de trabajo que se desempeñe; en el caso de las y los adolescentes, estos van de 30.00 a 90.00 pesos, hay una diminución que se justifica debido a la edad y no al trabajo que realizan: «le pagan 60.00 pesos porque es menor de edad» (Sr. R., 30 años, jornalero, febrero de 2012).

Los jefes y jefas de familia refirieron que sólo 15.5\% de sus hijos e hijas $(n=187)$ reciben una remuneración económica por su trabajo. Las edades de quienes reciben estos ingresos se encuentran entre 14 y 17 años, mientras que $50.3 \%$ no tiene un pago directo por su trabajo. Es el caso de niños y niñas menores de 14 años que no son reconocidos como trabajadores (as) formales, el $34.2 \%$ restante no recibe una remuneración económica porque supuestamente no trabaja.

Se encontraron casos de mujeres adolescentes de 15 años que mentían sobre su edad (la aumentaban a 17) porque ya realizaban, junto con su mamá y papá, actividades laborales como el descope (corte de las puntas de las matas de café) y el deshije (corte de ramas de las matas de café). Mentir sobre la edad era la única forma de acceder al pago y los beneficios que representa ser trabajador (a) de la finca (alimentación y vivienda). 
TABLA 1

Participación de la niñez en trabajos productivos por grupo de edad y sexo

\begin{tabular}{|c|c|c|c|c|c|}
\hline \multirow{2}{*}{ TRABAJOS PRODUCTIVOS } & \multicolumn{2}{|c|}{$\mathrm{EDAD}$} & \multirow{2}{*}{$\begin{array}{c}\text { MUJERES } \\
(\%)\end{array}$} & \multirow{2}{*}{$\begin{array}{c}\text { HOMBRES } \\
(\%)\end{array}$} & \multirow{2}{*}{ PAGO } \\
\hline & (AÑOS) & $\%$ & & & \\
\hline $\begin{array}{l}\text { Almácigo, cajete, llenado de } \\
\text { bolsa, escogida de café, deshi- } \\
\text { je, pepena y tapisca de café, } \\
\text { descope }\end{array}$ & 5 A 11 & 19.2 & 40.0 & 60.0 & $\begin{array}{l}\text { No reciben una remunera- } \\
\text { ción económica directa }\end{array}$ \\
\hline $\begin{array}{l}\text { Corte y limpia de cardamomo, } \\
\text { poda, chaporro, desombre }\end{array}$ & 12 a 17 & 57.5 & 30.4 & 69.6 & $\begin{array}{l}15.5 \% \text { recibe un pago que } \\
\text { va de los } 30.00 \text { a los } 90.00 \\
\text { pesos diarios. }\end{array}$ \\
\hline
\end{tabular}

Fuente: elaboración propia. Trabajo de campo, fincas cafetaleras, Chiapas, 2012.

\section{Trabajo reproductivo}

El trabajo reproductivo dentro las fincas cafetaleras es una práctica común, principalmente entre niños y niñas de 5 a 11 años de edad. Se pudo observar que $65.4 \%$ de las y los encuestados lo realizan. Entre los principales, se encuentran los domésticos dentro del hogar (23.1\%), el doméstico fuera del hogar (19.2\%), la combinación de ambos (7.7\%) y el cuidado de hermanos y hermanas (3.9\%). Se destaca que $11.5 \%$ realizan una doble jornada laboral. ${ }^{6}$

En el caso de niños, niñas y adolescentes de 12 a 17 años, sólo 30.0\% lo lleva a cabo. Existe un mayor número de mujeres realizando este tipo de trabajos. Sólo 13\% de los adolescentes varones se involucra en ellos. Las actividades que realizan los varones son aquellas que principalmente fortalecen su rol de proveedores del hogar, es decir, actividades relacionadas con el suministro de insumos (acarreo de leña y agua, por ejemplo).

Debido a que en el trabajo reproductivo de la infancia aparentemente no se involucran actores económicos como los administradores o los dueños de las fincas, éste suele ser poco contemplado y visibilizado como un problema,

${ }^{6}$ El $100 \%$ lo completan quienes refirieron que no trabajan (7.7\%) y aquellos (as) que no respondieron $(7.7 \%)$. 
incluso se observa como un trabajo fácil, que instruye y crea responsabilidades «benéficas» para el futuro de los infantes.

Como lo mencionan Carrasquer et al. (1998), Martínez et al. (2003) y Moreno (2007), hay una invisibilización y naturalización de este tipo de trabajos en las personas con ausencia de poder o autoridad dentro de los grupos domésticos, tal es el caso de los niños y niñas. Esta situación provoca el ocultamiento de su importancia para el desarrollo de las familias y las consecuencias que genera en el pleno desarrollo de quienes los realizan. Se encontraron hombres y mujeres que al responder sobre las actividades que efectuaban mujeres, niñas y niños, se expresaban de manera peyorativa diciendo: «sólo al hogar», «no hace nada, sólo los oficios de la casa».

\section{TABLA 2}

Participación de la niñez en trabajos reproductivos por grupo de edad y sexo

\begin{tabular}{|c|c|c|c|c|}
\hline \multirow{2}{*}{ TRABAJO REPRODUCTIVO } & \multicolumn{2}{|c|}{$\mathrm{EDAD}$} & \multirow{2}{*}{$\begin{array}{l}\text { MUJERES } \\
\%\end{array}$} & \multirow{2}{*}{$\begin{array}{l}\text { HOMBRES } \\
\%\end{array}$} \\
\hline & AÑOS & $\%$ & & \\
\hline Cuidado de niños y niñas más pequeños & 5 a 11 & 3.9 & 0.0 & 100.0 \\
\hline $\begin{array}{l}\text { Cuidado, aseo, alimentación y traslado de niños más } \\
\text { pequeños }\end{array}$ & 12 a 17 & 0.0 & 0.0 & 0.0 \\
\hline \multirow{2}{*}{$\begin{array}{l}\text { Trabajo doméstico dentro del hogar: Barrer, trapear, lim- } \\
\text { pieza de los espacios donde duermen, lavar trastes, lavar } \\
\text { ropa, cocinar, hacer tortillas, servir la comida }\end{array}$} & 5 a 11 & 23.1 & 50.0 & 50.0 \\
\hline & 12 a 17 & 15.0 & 100.0 & 0.0 \\
\hline $\begin{array}{l}\text { Trabajo doméstico fuera del hogar: Acarreo de agua, cor- } \\
\text { te y acarreo de leña, alimentación de animales domésti- } \\
\text { cos (perros, gallinas, guajolotes, patos y cerdos propiedad } \\
\text { de la familia, se habla en plural, sin embargo nunca son } \\
\text { más de uno por grupo doméstico), hacer mandados } \\
\text { (compra de alimentos, llevar mensajes, herramientas e } \\
\text { instrumentos de trabajo, comida), recoger alimentos }\end{array}$ & 12 a 17 & 19.2 & 60.0 & 100.0 \\
\hline
\end{tabular}

Fuente: elaboración propia. Trabajo de campo, fincas cafetaleras, Chiapas, 2012.

El trabajo reproductivo no es remunerado, sin embargo se observó su importancia cuando los hombres solteros o que no viajan con sus familias pagan a las adolescentes para que les laven la ropa o a los niños y niñas más pequeños para que les hagan mandados. Aunque el pago sea simbólico, sólo «para

$$
54 \frac{\text { PRIMER SEMESTRE } 2014}{\text { MIGRACIÓN Y DESARROLLO NÚM. } 22}
$$


sus chucherías» (Encargada de la guardería de la finca «I», febrero de 2012), destaca la importancia de la niñez en estos lugares, pues si viajaran con hijos e hijas propios (as) no tendrían que pagar por estos servicios.

En la asignación de los trabajos de tipo reproductivo dentro de las familias no se contemplan sus implicaciones futuras para la infancia, ni las repercusiones que pueden tener, si es que éste se convierte en su única posibilidad de vida. El trabajo infantil reproductivo es la puerta de entrada al ámbito laboral, pero también es un muro que impide otras expectativas, sueños y motivaciones. Los niños y niñas no son aprendices de estos trabajos, son responsables directos de su realización. Toman el lugar de las personas adultas y asumen los roles tradicionales de proveedores y cuidadoras del hogar, liberando a otros miembros de la familia para aumentar su participación en trabajos productivos.

\section{CONSIDERACIONES FINALES}

La migración laboral es una respuesta a los embates sociales y económicos del actual modelo de desarrollo, así lo sugieren $88.9 \%$ de hombres y mujeres guatemaltecos (as) que migran con el objetivo de mejorar sus condiciones económicas en las fincas cafetaleras del Soconusco, Chiapas. El fenómeno migratorio inserta a las y los individuos en un estado de vulnerabilidad social, pues van perdiendo la posibilidad de desarrollar capacidades que les permitan responder ante situaciones de alto riesgo o acceder a posibilidades que mejoren su calidad de vida.

Uno de los grupos más vulnerables es la infancia. Ésta se transforma en un conglomerado de seres invisibles, extranjeros, que se exponen permanentemente a la precarización laboral y exclusión social, sin que nadie se responsabilice de su bienestar por tratarse de un contexto transnacional.

La mayoría (79.7\%) de los adultos entrevistados dijeron que sus hijos e hijas apoyan más estando en las fincas que en sus lugares de origen. El 68.5\% de la niñez migrante realizan tanto trabajos productivos como reproductivos, que son distribuidos de acuerdo a su edad y sexo. Existe una división 
genérica del trabajo y por lo tanto una segmentación que coloca a las niñas y adolescentes mujeres en una posición de desventaja, pues la realización de trabajos productivos no las exime de la realización de trabajos reproductivos.

De quienes realizan trabajos productivos ( $60 \%$ de niños, niñas y adolescentes de 11 a 17 años y $30.7 \%$ de niños y niñas de 5 a 11), sólo 15.5\% recibe una remuneración económica directa. Los trabajos reproductivos no son reconocidos como trabajo, incluso se tiende a minimizar su utilidad a pesar de que es fundamental para el funcionamiento de las familias. Además, 65.4\% de niños y niñas de 5 a 11 años de edad y 30.0\% de adolescentes, en su mayoría mujeres, lo realizan. Se trata de un trabajo poco valorado que no genera ningún ingreso.

El trabajo productivo y reproductivo que realizan niños, niñas y adolescentes dentro de las fincas cafetaleras es una problemática social actual y vigente que debe ser observada como el resultado de profundos cambios globales, cuyas raíces son estructurales y en donde no es el individuo el responsable de su condición, sino más bien son fallas en los sistemas sociales, económicos, culturales y políticos los que determinan y posibilitan su persistencia. Se puede actuar, pero la estructura constriñe la acción individual y colectiva.

Ante dicha problemática son indispensables acciones que permitan la construcción de un andamiaje institucional eficiente, que garantice la protección universal de los mínimos de seguridad económica (trabajo e ingreso) y el bienestar social (servicios) que supone el pleno disfrute de los derechos de niñas y niños en condición migratoria.

\section{Bibliografía}

Anguiano Téllez, María Eugenia (2008), «Inmigración, emigración y tránsito migratorio en Chiapas: un bosquejo general», Liminar, Estudios Sociales y Humanísticos, vol. 6, núm. 2.

Barreiro García, Norma (1998), "El trabajo infantil un concepto de difícil consenso», en Norma Del Río Lugo (coord.), La infancia vulnerable de México en el mundo globalizado, UAm, Unicef, México.

$56 \frac{\text { PRIMER SEMESTRE } 2014}{\text { MIGRACIÓN Y DESARROLLO NÚM. } 22}$ 
Branka Lickic, Brboric y Carl Virik Schierup (2011), «Gobernabilidad asimétrica, trabajo decente y derechos de los migrantes», Migración y Desarrollo, vol. 9, núm. 17. Brasesco, Juan y Aída Nelly Mendoza (2011), "Construyendo territorios sin trabajo infantil», Rayuela, Revista Iberoamericana sobre Niñez y Juventud en lucha por sus Derechos, año 2, núm. 4.

Brizzio de la Hoz, Araceli (1996), El trabajo infantil en México, una realidad a superar, México, Instituto de Investigaciones Psicológicas de la Universidad Veracruzana, Unicef, ort.

(2002), «El trabajo infantil, una exclusión social», Foro invisibilidad y conciencia: Migración interna de niñas y niños jornaleros agrícolas en México, 26 y 27 de septiembre de 2002, http://www.uam.mx/cdi/pdf/eventos/invisibilidad/ trabajo_inf.pdf (consultado el 23 de mayo de 2012).

Carrasouer, Pilar, Teresa Torns, Elisabet Tejero y Alfonso Romero (1998), «El trabajo reproductivo», Papers Revista Sociológica, núm. 55, Barcelona, España.

CASIlLAS, Rodolfo (2010), Niños, niñas y adolescentes migrantes centroamericanos en poblaciones del sur de México, México, orm.

Castillo, Manuel Ángel (2006), Espacios diversos, historia en común, México, Secretaría de Relaciones Exteriores.

(2009), "Los derechos humanos de los inmigrantes en México», en Comisión Nacional de los Derechos Humanos (ed.), Prevención de la violencia, atención a grupos vulnerables y los derechos humanos. Los derechos de los migrantes, fascículo 5, México, CNDH.

Centro de Estudios de las Finanzas Públicas (cefp) (2001), El mercado del café en México, México, Cámara de Diputados.

Centro de Estudios Migratorios de la Unidad de Política Migratoria (Segob) (2012), "Extranjeros documentados para trabajar con una Tarjeta de Visitante Trabajador Fronterizo (TVTF), 1/ según sector de actividad, por país de residencia, entidad federativa y punto de expedición, 2012», http://www.inm.gob. $\mathrm{mx} /$ index.php/page/Documentacion_y_Legal_2012 (consultado el $10 \mathrm{de}$ enero del 2013).

(2012), «Extranjeros documentados para trabajar con una Tarjeta de Visitante Trabajador Fronterizo (TVTF), 1/ por entidad federativa, grandes grupos de edad y sexo, 2012», http://www.inm.gob.mx/index.php/page/ Documentacion_y_Legal_2012 (Consultado el 10 de enero del 2013).

Comisión Nacional de los Derechos Humanos (CNDH) (2009), Tríptico de Vulnerabilidad, México, CNDH. 
Cos Montiel, Francisco (1998), «Sirviendo a las mesas del mundo: las niñas y los niños jornaleros agrícolas en México», en Norma del Río Lugo (coord.), La infancia vulnerable de México en el mundo globalizado, México, UAM, Unicef.

Cruz Ángeles, Hugo y Martha Luz Rojas Wiesner (s/f), «Temas pendientes sobre la migración Internacional en la frontera sur de México», en Colegio de la Frontera Sur, Mesa V foro de migración, Temas pendientes sobre la migración internacional en la frontera sur de México, http://www.conapo.gob.mx/publicaciones/foronacional/mesa5.pdf (consultado el 28 de abril de 2011).

De Sousa Santos, Boaventura (2006), Conocer desde el Sur. Para una cultura política emancipatoria, Lima, Fondo Editorial de la Facultad de Ciencias Sociales, UnMSM, Programa de Estudios sobre Democracia y Transformación Global.

Delson Atalaya, Ana (2003), «La división entre público y privado como eje estructurador de la desigualdad de género", en Adela García (coord.), Género en la cooperación al desarrollo, una mirada al desarrollo, Madrid, España, ACSUR-Las Segovias.

Encuesta Nacional de Condiciones de Vida (encovi) (2011), "Pobreza y desarrollo», http://www.ine.gob.gt/np/encovi/encovi2011.htm (consultado el 10 de enero de 2013).

Feldmann, Andreas y Jorge Durand (2008), «Mortandad en la frontera», Migración y Desarrollo, vol. 10, núm. 10.

Galeana Cisneros, Rosaura (2000), "El trabajo infantil en México balance de una década", en Colectivo de apoyo a la niñez (ed.), Avances y retrocesos: Balance de una década. V Informe sobre los Derechos y la Situación de la Niñez en México 1998-2000, México, COMEXANi.

George, Susan (2003), «żGlobalización de los derechos?», en Matthew J. Gibney (ed.), La globalización de los derechos humanos, Barcelona, Crítica.

Girón, Carol (2010), «Migrantes Mam entre San Marcos (Guatemala) y Chiapas (México)», en P. Alicia Torres (coord.), Niñez indígena y migración. Derechos en riesgo y tramas culturales, Quito, Flacso, AECID, Unicef.

GloKner FragetTI, Valentina (2010), «Explotación Infantil Jornalera y capitalismo postfordista», Revista Internacional NAT's, núm. 18.

GzesH, Susan (2008), «Una redefinición de la migración forzosa con base en los derechos humanos", Migración y Desarrollo, vol. 10, núm. 10.

Instituto para la Seguridad y la Democracia (Insyde) 2008, «Percepción sobre la migración en la Frontera Sur. Encuesta en vivienda, Tapachula, Chiapas», http://insyde.org.mx/images/reporte\%20 percepciones $\% 20$ sobre $\% 20$

$58 \frac{\text { PRIMER SEMESTRE } 2014}{\text { MIGRACIÓN Y DESARROLLO NÚM. } 22}$ 
la\%20migraci\%C3\%B3n\%20en\%20la\%20frontera\%20sur.pdf (consultado el 2 de enero de 2012).

Krichesky, Marcelo David (1992), Trabajo infantil y escolaridad primaria, Argentina, Flacso.

Ley Federal del Trabajo, Última reforma publicada: DoF 30-11-2012, http://www. diputados.gob.mx/LeyesBiblio/pdf/125.pdf (consultado el 7 de enero de 2013).

Liwski, Norberto Ignacio (2012), «Migración de niñas, niños y adolescentes bajo el enfoque de los derechos", Rayuela, Revista Iberoamericana sobre Niñez y Juventud en la Lucha por sus Derechos. Niñez y Migración, año 3, núm. 5.

López ZúñIGA, Noé (2011), La migración bajo la óptica del derecho, México, Miguel Ángel Porrúa.

Márouez Covarrubias, Humberto y Raúl Delgado Wise (2011), «Una perspectiva del sur sobre capital global, migración forzada y desarrollo alternativo», Migración y Desarrollo, vol. 9, núm. 16.

Martínez Corona, Beatriz, Silvia Martínez, Socorro Barrientos y Alberto Paredes (2003), «Mujeres Rurales y género. Aportes para el diseño de políticas públicas», en Beatriz Martínez Corona y Rufino Díaz Cervantes (coords.), Mujeres rurales, género, trabajo y transformaciones sociales, México, Colpos Campus Puebla, Conacyt, IPM.

Moreno Ruíz, María (2007), "Políticas conciliatorias entre los ámbitos productivo y reproductivo en América Latina: una cuestión estratégica para abordar la desigualdad», en Gisela Zaremberg (coord.), Políticas sociales y género, Tomo II, Los problemas sociales y metodológicos, México, Flacso.

Organización de las Naciones Unidas para la Agricultura y la Alimentación (fao) (2011), «Panorámica económica, social y san de Guatemala, 2011», http://coin. fao.org/cms/world/guatemala/InformaccionSobreElPais/Panoramasan2011. html (consultado el 6 de enero de 2013).

Organización Internacional para las Migraciones (oim), Fondo de las Naciones Unidas para la Infancia (Unicef) (2010), Remesas 2010, niñez y adolescencia, Guatemala.

Palma C., Silvia Irene (2007), Migración en la época de post-conflicto: Vulneración de derechos de las poblaciones excluidas e impacto sobre la participación política, Guatemala, Consejería de Proyectos.

Pogge, Thomas (2007), «Reconocidos y violados por la ley universal: los derechos humanos de los pobres globales», en Francisco Cortés Rodas y Miguel Giusti, 
Justicia global, derechos humanos y responsabilidad, Bogotá, Siglo del Hombre Editores.

Procurador de los Derechos Humanos (PdH) (2011), Informe Anual Circunstanciado: Resumen ejecutivo del informe Anual Circunstanciado al Congreso de la República de las actividades y de la situación de los derechos humanos en Guatemala durante el 2011, Guatemala, Procurador de los Derechos Humanos.

Rojas Rangel, Teresa (2006), «Las niñas y los niños jornaleros migrantes en México: condiciones de vida y trabajo", ponencia presentada en III Conferencia de la Red Latinoamericana y del Caribe de Childwatch International, http://sociedadlatinoamericana.bligoo.com/las-ninas-y-los-ninos-jornaleros-migrantes-en-mexicocondiciones-de-vida-y-trabajo (consultado el 25 de marzo de 2012).

(2012), «Migración y ocupación en la fuerza de trabajo infantil en regiones agroexportadoras", Rayuela. Revista Iberoamericana sobre Niñez y Juventud en la Lucha por sus Derechos. Niñez y Migración, año 3, núm. 5.

SaAdeh Rivera, Ana Mirella (2011), "Niñez y adolescencia trabajadora: un asunto de doble moral», Rayuela. Revista Iberoamericana sobre Niñez y Juventud en lucha por sus Derechos. Niñez y trabajo, enfoques disensos-consensos, año 2, núm. 4.

Salgado de Snyder, V. Nelly, Tonatiuh González Vázquez, Ietza Bojorquez Chapela y César Infante Xibille (2007), «Vulnerabilidad social, salud y migración MéxicoEstados Unidos», Salud pública de México, vol. 49, México, Instituto Nacional de Salud Pública.

Secretaría de Agricultura, Ganadería, Desarrollo Rural, Pesca y Alimentación (Sagarpa) (2012), "Alcanza precios récord café de México de alta calidad», http://www.sagarpa.gob.mx/saladeprensa/boletines2/Paginas/2012B363. aspx (consultado el 14 de septiembres de 2012).

SuÁrez San Román, Blanca, Emma Zapata Martelo, Rosario Ayala Carrillo, Naima Cárcamo Toalá y Josefina Manjarrez Rosas (2011), iY las mujeres rurales? Avances y desafíos en las políticas públicas, México, Indesol, GIMTAP.

Todaro, Rosa y Sonia Yáñez (eds.) (2004), El trabajo se transforma. Relaciones de producción y relaciones de género, Chile, Ediciones CEM.

Unicef (2010), Guatemala, la tormenta perfecta. Impacto del cambio climático y la crisis económica de la niñez y la adolescencia, Guatemala, Unicef.

Zapata MarTelo, Emma y Blanca Suárez San Román (2012), «Migración: reasignación de roles en espacios locales y transnacionales», Ra Ximhai, vol. 8, núm. 1. 